\title{
Des mémoires « irréconciliables » du Rwanda au sein de l'armée française ?
}

\section{Le rapport Duclert et les multiples voix de « la grande muette »}

\author{
Étienne Smith
}

Citer cet article : Smith Étienne (2021), « Des mémoires "irréconciliables" du Rwanda au sein de l'armée française ? Le rapport Duclert et les multiples voix de "la grande muette" », Revue d'Histoire Contemporaine de I'Afrique, Dossier : au-delà du rapport Duclert, 52-63, en ligne. URL :

https://oap.unige.ch/journals/rhca/article/view/rwandasmith

Mise en ligne : 08 novembre 2021

DOI : https://doi.org/10.51185/journals/rhca.2021.e580

\section{Résumé}

Cet article propose une brève analyse de la façon dont le rapport Duclert décortique l'implication militaire française au Rwanda entre 1990 et 1994. Comme le rapport le met bien en lumière, cet engagement est, dès le départ, traversé de tensions. La mémoire de ces opérations militaires au sein de l'institution ne fera pas non plus l'objet d'un consensus malgré les tentatives de l'association France Turquoise d'imposer un récit univoque. Après la porte entrouverte par la mission d'information parlementaire en 1998, le rapport marque une rupture en donnant une caution institutionnelle à des voix moins médiatisées, mais plus lucides, dans les armées. On pourra regretter cependant que la commission Duclert n'ait pas su, ou pu, approfondir certains aspects, comme les relations sur le terrain entre officiers français et officiers des Forces armées rwandaises, en particulier la fabrique du renseignement ou le recours éventuel à une stratégie indirecte plus discrète.

Mots-clés : Rwanda ; génocide ; rapport Duclert ; armée française 
Cela a été dit et redit : le rapport Duclert ne vient pas bouleverser nos connaissances sur I'engagement français au Rwanda entre 1990 et 19941. II n'apporte pas de faits majeurs nouveaux, en tout cas pas de « révélations ». L'essentiel avait déjà été mis en lumière lors de la mission d'information parlementaire (MIP) de 1998², de la commission d'enquête citoyenne de $2004^{3}$, et dans plusieurs ouvrages de militants et de journalistes ${ }^{4}$. Le rapport Duclert vient néanmoins caractériser l'engagement avec plus de précision que ne l'a fait la MIP dont les conclusions venaient euphémiser une partie de son contenu particulièrement riche ${ }^{5}$. Le rapport Duclert, lui, déroule les conséquences de l'intervention française avec minutie et pointe plus directement les responsabilités du président Mitterrand, de l'entourage présidentiel et en particulier de l'état-major particulier. Il a surtout permis, et c'est ce qu'on retiendra ici, de libérer la parole au sein de l'armée, parole qui avait été jusque-là monopolisée, voire confisquée, par certains protagonistes arc-boutés sur la défense coûte que coûte du rôle de la France, réunis en particulier au sein de l'association France-Turquoise. Plus encore, le rapport souligne la pluralité des analyses au sein de la "grande muette », dès cette période, et ce contrairement à l'image monolithique ou consensuelle que certains politiques ou certains responsables militaires ont voulu donner de l'engagement français au Rwanda. Au total, le rapport Duclert permet d'assumer une mémoire de l'événement qui reconnaît des fautes et des responsabilités accablantes, sans être suspect d'antimilitarisme, et sans pour autant endosser les accusations de complicité de génocide que le rapport a écarté de façon assez définitive. En revenant sur l'évolution des prises de positions de militaires français depuis 1994, comme on se le propose ici, on peut mesurer la nouvelle étape franchie par la publication de ce rapport, même si des aspects concrets de l'engagement militaire français sur le terrain restent encore largement à

\footnotetext{
${ }^{1}$ Voir notamment Robinet François (2021), « Rwanda 1994 : un rapport pour l'histoire ? ", Études, juillet-août, pp. 7-18 ; Vallée Shahin (2021), «Rapport Duclert sur le génocide des Tutsi: un pas de plus», Le Grand Continent, 2 avril, en ligne. URL: https://legrandcontinent.eu/fr/2021/04/02/rapport-duclert-sur-le-rwanda-un-pas-de-plus/ (consulté le 11 octobre 2021); Samuel Kuhn, « France-Rwanda, retour sur un dossier brûlant [2] : rapports et recherches. Entretien avec Stéphane Audoin-Rouzeau et François Graner », Fragments sur les temps présents, 28 juillet 2021, en ligne. URL : https://tempspresents.com/2021/07/28/france-rwandaretour-sur-un-dossier-brulant-2-rapports-et-recherches/ (consulté le 11 octobre 2021); Jean-François Bayart, "Rwanda: la restauration autoritaire au prix d'un génocide », AOC, 5 mai 2021, en ligne. URL : https://aoc.media/opinion/2021/05/04/rwanda-larestauration-autoritaire-au-prix-dun-genocide/ (consulté le 11 octobre 2021)

2 « Rapport d'information déposé par la mission d'information de la commission de la Défense nationale et des Forces armées et de la commission des Affaires étrangères, sur les opérations militaires menées par la France, d'autres pays et l'Onu au Rwanda entre 1990 et $1994 », n^{\circ} 1271$, Paris, Assemblée nationale, 15 décembre 1998

${ }^{3}$ Coret Laure et Verschave François-Xavier (dir.) (2005), L'Horreur qui nous prend au visage : I'État français et le génocide au Rwanda. Rapport de la Commission d'enquête citoyenne, Paris, Karthala.

${ }^{4}$ Ce sont en effet plus des journalistes ou des militants associatifs qui se sont saisis de la question quand la recherche semblait absente. Parmi une trentaine de livres, et pour se limiter aux ouvrages en français, voir entre autres, chronologiquement, Mas Monique (1999), Paris-Kigali 1990-1994. Lunettes coloniales, politique du sabre et onction humanitaire pour un génocide en Afrique, Paris/Montréal, L'Harmattan ; Saint-Exupéry Patrick (de) (2004), L'Inavouable. La France au Rwanda, Paris, Les Arènes ; Vulpian Laure (de) et Prungnaud Thierry (2012), Silence Turquoise. Rwanda 1992-1994, responsabilités de l'État français dans le génocide des Tutsi, Paris, Don Quichotte ; Collombat Benoît et Servenay David (2014), « Au nom de la France ». Guerres secrètes au Rwanda, Paris, La Découverte; Graner François (2014), Le Sabre et la machette: officiers français et génocide tutsi, Bruxelles, Éditions Tribord Dupaquier Jean-François (2014), Politiques, militaires et mercenaires français au Rwanda. Chronique d'une désinformation, Paris, Karthala ; Larcher Laurent (2019), Rwanda, ils parlent. Témoignages pour l'histoire, Paris Seuil ; Doridant Raphaël et Graner François (2020), L'État français et le génocide des Tutsis au Rwanda, Marseille, Agone. La recherche " académique », en revanche, s'est peu penchée sur la question de l'engagement français. Voir Ambrosetti David (2001), La France au Rwanda. Un discours de légitimation morale, Paris, Karthala; Lanotte Olivier (2007), La France au Rwanda (1990-1994). Entre abstention impossible et engagement ambivalent, Bruxelles, Peter Lang, ainsi que le dossier de la revue Esprit, «France-Rwanda, et maintenant ? », mai 2010, pp. 80-171. ${ }^{5}$ Voir en particulier les documents rendus publics, certaines auditions et les annexes toujours disponibles sur le site de l'Assemblée nationale. URL : https://www.assemblee-nationale.fr/dossiers/rwanda/rapport.asp (consulté le 11 octobre 2021).
} 
étudier et si des oppositions au rapport Duclert persistent chez certains acteurs clés de l'époque.

\section{Des fissures dans le monolithe}

Cette libération de la parole se mesure aux nombres de tribunes de militaires parues depuis la publication du rapport Duclert pour en appuyer les conclusions ${ }^{6}$. En 1998, déjà, trente militaires français avaient été auditionnés par les parlementaires, mais seuls onze comptes-rendus d'audition avaient été publiés. Pour le lecteur attentif, les dissensions se révélaient déjà, mais à mots couverts, car les mémoires sont encore vives et les protagonistes souvent encore en fonction. Avec le reflux de l'attention médiatique après 1998', les divergences d'appréciation même feutrées n'apparaissent plus publiquement et l'on ne semble plus trop parler du Rwanda dans les armées. II faut attendre les accusations lancées par plusieurs associations contre des militaires français à partir de $2004^{8}$ pour que d'anciens officiers prennent la plume, pour tenter de défendre leur rôle lors de l'opération Turquoise notamment. Toutes ces prises de parole vont dans le même sens: une défense, avec plus ou moins de nuances, de l'engagement français ${ }^{9}$, à quelques exceptions près ${ }^{10}$. Plus récemment, l'opération Noroît (1990-1993) a également fait l'objet d'un livre, soulignant combien l'engagement militaire français au Rwanda continue de susciter des tentatives de clarifications ${ }^{11}$. II n'y a que l'opération Amaryllis (avril 1994) qui n'ait pas encore son ouvrage.

Si donc l'immense majorité de la production écrite militaire sur les OPEX (opérations extérieures) françaises au Rwanda s'inscrivait jusque-là dans une volonté de défendre leur

\footnotetext{
${ }^{6}$ Piotr Smolar, « Pour le général Jean Varret, le rapport Duclert sur le Rwanda permet de "sortir de vingt-six ans de débats stériles" », Le Monde, 29 mars 2021, en ligne. URL : https://www.lemonde.fr/international/article/2021/03/29/le-general-jean-varret-le-rapportduclert-sur-le-rwanda-permet-de-sortir-de-vingt-six-ans-de-debats-steriles_6074803_3210.html (consulté le 11 octobre 2021) ; Patrice Sartre, «Le rapport Duclert rend justice aux soldats de l'opération Turquoise », Le Monde, 30 mars 2021, en ligne. URL: https://www.lemonde.fr/idees/article/2021/03/30/general-patrice-sartre-sur-le-rwanda-le-rapport-duclert-rend-justice-aux-soldatsde-I-operation-turquoise_6074951_3232.html (consulté le 11 octobre 2021); "René Galinié : J'ai dit: "attention on va au massacre!" ", AFP, 31 mars 2021

7 Pour un retour sur la saillance à éclipses du Rwanda dans l'espace public plus généralement entre 1994 et 2014 , voir Robinet François (2014), «L'empreinte des récits médiatiques : mémoires françaises du génocide des Tutsi du Rwanda », Les Temps modernes, 680-681, pp. 166-188. Sur les enjeux et instrumentalisations autour du rôle de la France, voir Chrétien Jean-Pierre (2009), « France et Rwanda : le cercle vicieux », Politique africaine, 113, pp. 121-137 et Robinet François (2021), "Le rôle de la France au Rwanda : I'histoire piégée ? ", Revue d'histoire culturelle. XVIIF-XXF siècles, 2, en ligne. URL : https://revues.mshparisnord.fr/rhc/index.php?id=690 (consulté le 11 octobre 2021)

8 En 2004, des plaintes pour viols, et en 2005 pour " complicité de génocide et complicité de crimes contre l'humanité », ont été déposée par les associations Survie, la Fédération internationale pour les droits humains (FIDH) et la Ligue des droits de I'homme $(\mathrm{LDH})$ notamment

9 Hogard Jacques (2005), Les Larmes de I'honneur. 60 jours dans la tourmente du Rwanda, Paris, Hugo Doc ; Tauzin Didier (2011), Rwanda : je demande justice pour la France et ses soldats. Le chef de l'opération Chimère témoigne, Paris, Jacob Duvernet ; Lafourcade Jean-Claude et Riffaud Guillaume (2010), Opération Turquoise. Rwanda 1994, Paris, Perrin. Sur ces ouvrages apologétiques, en particulier les deux premiers qui se distinguent par leur extrémisme, voir Smith Étienne (2014), "Les derniers défenseurs de l'empire : quand l'armée française raconte ses Rwanda », Les Temps modernes, 680-681, pp. 66-100.

10 Voir le témoignage du capitaine Ancel Guillaume (2018), Rwanda, la fin du silence. Témoignage d'un officier français, Paris, Les Belles Lettres, dans lequel l'auteur souligne l'ambiguïté initiale offensive de l'opération Turquoise pour l'avoir vécue en devant accompagner des frappes aériennes contre le Front patriotique rwandais (FPR), annulées in extremis et sans explications. Voir aussi Vulpian L. (de) et Prungnaud T., Silence Turquoise..., op. cit.

11 Delort Dominique (2021), Guerre au Rwanda. L'espoir brisé, 1991-1994, Paris, Perrin/ Pierre de Taillac. Voir également le témoignage du colonel Michel Goya, "Je suis complice de génocide mais je me soigne », blog La Voie de l'épée, 6 juillet 2017, en ligne. URL: https://lavoiedelepee.blogspot.com/2017/07/je-suis-complice-de-genocide-mais-je-me.html (consulté le 11 octobre 2021). Pour une lecture plus critique de cette période 1990-1993, voir le chapitre consacré au Rwanda dans le livre du général Varret Jean (2018), Général, j'en ai pris pour mon grade, Paris, Éditions Sydney Laurent.
} 
légitimité, l'autorité institutionnelle du rapport Duclert, introduit clairement une rupture. II vient faciliter la prise de parole publique de voix plus diverses au sein des armées et par définition non suspectes d'antimilitarisme. Il devient enfin possible pour des militaires d'assumer publiquement des positions alternatives au récit figé et verrouillé de l'engagement français imposé par les généraux Quesnot et Huchon et relayé dans l'espace public notamment par Pierre Péan, Hubert Védrine et l'association France-Turquoise.

Le rapport Duclert retrace bien l'enjeu qu'a constitué, dès 1990, la définition d'une position sur l'engagement militaire français au Rwanda. Tout d'abord des hésitations, des sons de cloche différents, puis comment, petit à petit, une position maximaliste a pu s'imposer et s'enferrer dans un engrenage fatal en sélectionnant et encourageant l'information conforme, en marginalisant les grilles de lecture alternatives y compris celles au sein de ses administrations. L'état-major des armées et la mission militaire de coopération sont initialement prudents. Mais progressivement, l'état-major particulier du président (EMP), les troupes de marines et les forces spéciales font prévaloir leurs vues, imposent leurs hommes et leurs méthodes. Le $1^{\text {er }}$ RPIMa (Régiment de parachutistes d'infanterie de marine) devient la cheville ouvrière de la guerre française au Rwanda, ses héritages coloniaux et de la guerre froide en Afrique en deviennent les grilles de lecture. Son réseau mène aussi aux postes clés ${ }^{12}$.

La mainmise ne se fait toutefois pas sans résistances. En effet, au sein de l'appareil politico-militaire français, malgré l'importance géostratégique toute relative de l'enjeu rwandais au regard de la guerre du Golfe puis de la guerre en ex-Yougoslavie, les tensions sont parfois vives. Dans les ministères comme sur le terrain, les lignes de clivage au fond assez classiques entre "faucons » et "modérés » ne manquent pas, et c'est un des mérites du rapport Duclert que de rappeler ces conflits souvent effacés par la défense « en bloc » de «I'honneur » de l'armée. Au printemps 1991, l'attaché de défense René Galinié s'oppose par exemple aux préconisations du lieutenant-colonel Canovas concernant l'élargissement massif du recrutement des Forces armées rwandaises (FAR) selon les lignes voulues par la partie rwandaise $^{13}$. Le rapport note également qu'en avril 1992 la nomination d'un conseiller français du chef d'état-major (CEM) des FAR fait l'objet d'un conflit entre armée de terre (dont font partie les troupes de marine) et coopération ${ }^{14}$. Le colonel Huchon, adjoint à I'EMP, parvient à imposer son homme, le lieutenant-colonel Jean-Jacques Maurin, issu du $1^{\text {er }}$ RPIMa. Sur place, selon le rapport, ce dernier s'entend bien avec le général Rosier ( $1^{\text {er }}$ RPIMa également) mais est en conflit avec l'attaché de défense, issu de la gendarmerie, Bernard Cussac. Difficile de ne pas y voir des conflits entre troupes de marine et gendarmerie, toujours d'après le rapport. En 1993, le conflit entre le chef de la mission de coopération, le général Jean Varret, et le colonel Huchon s'envenime. Le premier réalise que le second court-circuite les canaux hiérarchiques classiques et soustraie le Dami (dispositif d'assistance militaire) à son autorité ${ }^{15}$. Devenu général, Huchon le remplacera à son poste, assurant une mainmise de I'EMP sur le dossier

\footnotetext{
12 Les officiers Jean-Pierre Huchon, Gilbert Canovas, Jacques Rosier, Jean-Jacques Maurin, Didier Tauzin, Gilles Chollet, entre autres, ont commandé ou sont issus du $1^{\text {er }}$ RPIMa.

${ }^{13}$ Commission de recherche sur les archives françaises relatives au Rwanda et au génocide des Tutsi (2021), La France, le Rwanda et le génocide des Tutsi (1990-1994), Paris, Armand Colin, p. 898. Ci-après Rapport Duclert.

14 Rapport Duclert, pp. 156-157.

15 Rappelé au ministère de la Défense, le général Varret démissionne suite à ce désaveu qu'il impute à sa volonté de rester conforme aux consignes de neutralité de l'engagement français, quand ce qu'il qualifie de « lobby militaire » tend au contraire à l'engagement direct.
} 
Rwanda. Tout ceci confirme que ce dossier est piloté au plus haut niveau à l'Élysée, que l'EMP s'assure d'une certaine verticalité du commandement sur la question en plaçant ses hommes et en exigeant des retours d'information directs au-delà des canaux classiques. Entre-temps, en 1992, la création du commandement des opérations spéciales (COS) facilite l'emploi des forces spéciales en raccourcissant la chaîne de commandement.

L'opération Turquoise en juin-juillet 1994 donnera lieu à de nouvelles passes d'armes entre faucons et modérés sur les objectifs de l'opération, décuplées par l'avènement de la seconde cohabitation qui complique encore plus les clivages ${ }^{16}$. Sur le terrain, la confrontation au génocide a aussi produit de nouvelles lignes de fracture, entre ceux qui connaissaient le terrain, qui avaient «fait » le Rwanda avant 1994, et les autres. Les nouveaux venus ont pu poser un regard neuf et parfois (se) poser des questions sur les objectifs de l'opération, sur l'ambiguïté des consignes initialement reçues ${ }^{17}$. À l'inverse, les routines d'action et de pensée des seconds, alimentées par les œillères de la «frère d'armitude » avec les FAR, ont agi comme autant de biais de confirmation en faveur d'une belligérance accrue avec le FPR et une accointance plus marquée avec l'état-major des $\mathrm{FAR}^{18}$.

Pour éclairer ces diverses lignes de fractures, les clivages corporatistes ou idéologiques traditionnellement mobilisés pour expliquer des dissensions dans les armées peuvent constituer une toile de fond des incompréhensions réciproques. On pense aux polarisations entre corps d'armées, entre régiments, entre catholiques traditionalistes et francs-maçons, entre «atlantistes » et « souverainistes » ou entre «libéraux » et « nationalistes », entre militaires « européens » polyglottes habitués des opérations multilatérales, de l'Onu et l'Otan et militaires ayant fait l'essentiel de leur carrière dans la défense du pré carré françafricain (troupes de marine et légion étrangère). II faudrait ajouter enfin les clivages selon le niveau de formation intellectuelle et le désir d'action, entre " officiers de salon » et « officiers de terrain », entre «combattants » et "technocrates ${ }^{19}$. Mais ces clivages ne semblent fonctionner que partiellement ici. II s'agit d'abord et surtout d'une question d'éthique individuelle sur l'arbitrage entre objectifs militaires et droits de l'homme dans des situations de coopération militaire et alors que des massacres ont déjà eu lieu (1990-1993). Même si les socialisations corporatistes

\footnotetext{
16 Sur les débats à Paris au ministère de la Défense, voir Rapport Duclert, pp. 447-463 et Prunier Gérard ([1995] 1999), Rwanda : /e génocide, Paris, Dagorno, p. 348. Le conseiller de Matignon, Philippe Baudillon, semble avoir fait les frais de ces conflits pour avoir annoncé au FPR qu'une " nouvelle politique » africaine était à l'œuvre avec le nouveau gouvernement de cohabitation.

17 Ce ne fut pas le cas de tous les "nouveaux venus », comme le responsable du groupement Sud de Turquoise, Jacques Hogard, dont la commission Duclert signale la différence de tonalité auto-satisfaite de son rapport de fin de mission avec celui du responsable du groupement Nord, Patrice Sartre, beaucoup plus lucide. Voir Rapport Duclert, note 627, pp. 1188-1889.

18 Pour trouver trace de ces antagonismes qui ont émaillé l'opération Turquoise sur le terrain, on peut se replonger dans les reportages des nombreux journalistes ayant suivi l'opération. Jean d'Ormesson, pourtant étroitement cornaqué par l'officier responsable de la communication de Turquoise, soulignait les « opinions assez divergentes, au sein même des forces françaises à l'égard du FPR » dans ses reportages pour Le Figaro du 19 au 21 juillet 1994. Fait intéressant, cette osmose avec les FAR de certains " anciens » voulant volontiers reconquérir Kigali, ne les a pas rendus plus à l'aise avec les objectifs de Turquoise puisque précisément, comme les FAR, certains en attendaient plus de l'opération et seront aussi déçus par les objectifs finalement limités de Turquoise. Ils devront même pour certains, comme le colonel Tauzin, être recadrés, pour mettre fin à la cacophonie dans la presse sur les objectifs de I'opération. Voir aussi le témoignage du chef de la Mission des Nations unies pour l'assistance au Rwanda (Minuar), Dallaire Roméo (2003), J'ai serré la main du diable. La faillite de l'humanité au Rwanda, Outremont (Québec), Libre Expression, pp. 559-560, 569, qui distingue le commandement de Turquoise et certains officiers français extrémistes.

19 Une large littérature académique existe sur la sociologie de l'institution militaire et de ses mutations (on pense ici aux travaux de Gregory Daho, Cristel Coton, Jean Joana et Fabrice Hamelin entre autres), que le rapport a choisi de ne pas mobiliser, alors qu'ils auraient tous permis d'enrichir l'analyse du corpus. Cela semble devoir s'expliquer par la volonté de la commission de se focaliser uniquement sur les archives administratives. En sens inverse, on peut déplorer que cette littérature "généraliste » sur l'institution militaire ne se soit pas intéressée à l'engagement militaire français au Rwanda, malgré son importance et son héritage de controverses.
} 
jouent, il s'agit aussi de dilemmes au fond très personnels qui surgissent dans la confrontation intime au mal absolu qu'est un génocide (Amaryllis et Turquoise). Cette expérience peut être l'objet de remises en causes profondes, du début d'une introspection et d'un malaise, des premiers cheveux blancs, ou plutôt de refoulement voire de déni au profit d'autres priorités stratégiques et opérationnelles. La gamme de réactions diverses des militaires français témoigne de ces infinies variations à hauteur d'homme.

On est frappé, à cet égard, par les nombreux silences, ou absences, de cet univers des archives administratives ${ }^{20}$. Du côté des sous-officiers ou soldats du rang, le panel des sensibilités et réactions face au choc qu'a été le génocide des Tutsi a aussi été large, mais totalement invisible, en tout cas inaudible dans l'espace public: malaise, refoulement, on l'a dit, mais aussi stress post-traumatique, dépression, alcoolisme, ruptures familiales. Des «pleurs » même à la simple évocation du Rwanda chez des anciens de Turquoise ou d'Amaryllis. Quand on interroge un militaire, le Rwanda est généralement, de toutes les opérations ou de concert avec la Bosnie, celle qui a le plus marqué en raison de son contexte si particulier ${ }^{21}$. Le besoin de suivi psychologique, les nombreux stress post-traumatiques bien particuliers, non pas liés à des épisodes de combats comme en Afghanistan ou au Mali par exemple (il n'y en a quasiment pas eu pendant Turquoise), mais bien nés de la confrontation à l'indicible de l'extermination ${ }^{22}$. Toute l'histoire de ce vécu "par le bas » d'une OPEX si particulière comme Turquoise est donc encore à faire.

Retracer les dissensus, les rapports de force, les échecs, l'orientation de l'information, les «blocages cognitifs", ce n'est pas porter atteinte à l'honneur d'une opération comme Turquoise et ceux qui l'ont mené à différents échelons. Comme le confirme le rapport Duclert, c'est au contraire en avoir une lecture plus réaliste, au-delà des récits idéologiques qui en sont faits, et mesurer combien l'attitude individuelle et le positionnement de chacun est la clé de lecture la plus intéressante et la plus fine pour comprendre les événements qui ont fait polémique, comme l'assistance tardive aux rescapés de Bisesero, les relations avec les autorités génocidaires ou l'attitude face au FPR.

\section{L'engagement militaire franco-rwandais au concret : une histoire encore à écrire}

Si le rapport Duclert éclaire d'une lumière sans concessions les dérives de certains acteurs au sommet de l'État, le concret de l'engagement franco-rwandais sur le terrain reste encore

\footnotetext{
20 Les riches archives audiovisuelles utilisées partiellement par le rapport sont néanmoins à signaler et à explorer davantage.

21 Le général Lafourcade témoigne qu'il lui fallut dépêcher en urgence trois psychiatres depuis Paris pendant I'opération Turquoise pour près d'une cinquantaine de cas nécessitant un suivi. Voir Leila Minano et Julia Pascual, «Viols au Rwanda. De nouvelles victimes accusent les soldats français ", Causette, novembre 2011, p. 69.

22 Les récits d'expérience sont nécessairement différents, et leur incommensurabilité participe parfois aux malentendus. Ainsi, ceux des militaires français exclusivement déployés sur le territoire zaïrois n'auront pas éprouvé de visu le génocide, et se référeront dans leurs récits surtout aux flux de réfugiés hutu à Goma et à la gestion de de l'épidémie de choléra, alors que ceux déployés au Rwanda auront été confrontés aux traces récentes de l'extermination des Tutsi.
} 
largement à explorer ${ }^{23}$. Malgré les apports de la MIP et de certains ouvrages ${ }^{24}$, I'action des conseillers militaires français auprès de leurs homologues rwandais reste insuffisamment documentée. Ces conseillers ont notamment servi au sein d'unités d'élite comme la garde présidentielle, les para-commandos et le bataillon de reconnaissance, qui se sont ensuite distinguées par leur rôle actif dès le début du génocide. Pour ce volet concret de la coopération militaire, les archives rwandaises et le recours aux témoignages français et rwandais sont indispensables. Fin décembre 1993, on compte ainsi 89 officiers et sous-officiers français dans I'assistance technique militaire. Leurs souvenirs mériteraient d'être entendus, au-delà des seuls rapports officiels. Mais ceux de la partie rwandaise aussi, au risque d'écrire uniquement la moitié de l'histoire de cette coopération. Le contraste est en effet toujours frappant, entre le discours officiel français sur le rôle des coopérants s'en tenant aux consignes de neutralité, et les témoignages sur le prestige, le rôle important voire décisif des coopérants au sein des unités qu'ils «conseillent $»^{25}$. Vaste chantier assurément que cette histoire et sociologie de la coopération militaire française, au Rwanda, et en Afrique plus généralement ${ }^{26}$.

Les liens noués avec des officiers des FAR, y compris les plus extrémistes, sont trop peu explorés pour mesurer les effets de l'intoxication réciproque d'extrémistes rwandais et d'officiers français dont certains vont développer une osmose idéologique avec ces ultras du Hutu Power. Qui intoxique qui ? Qui radicalise qui ? Qui incite l'autre à ne faire aucune concession au FPR? Qui influence l'autre sur la façon de considérer l'opposition hutu démocrate comme faible voire « traître » à l'effort de guerre ? La réponse à ces questions n'est pas aisée. Le lieutenant-colonel Maurin à lui tout seul symbolise ces attachements qui posent question. Bien que d'abord conseiller du CEM Déogratias Nsabimana d'avril 1992 à avril 1994, il est en excellents termes avec des ultras rwandais comme Laurent Serubuga, Théoneste Bagosora ou Anatole Nsengiyumva ${ }^{27}$. De son côté, le colonel Didier Tauzin, ne tarit pas d'éloges sur certains officiers des FAR, comme Augustin Bizimungu, sans préciser que ce dernier sera condamné par le Tribunal pénal international pour le Rwanda (TPIR) à trente ans de prison pour génocide, crimes contre l'humanité et crimes de guerre ${ }^{28}$.

\footnotetext{
23 Hélène Dumas, «Rwanda-France : au plus près de la matérialité du génocide des Tutsi », AOC, 11 mars 2021, en ligne. URL: https://aoc.media/analyse/2021/03/10/rwanda-france-au-plus-pres-de-la-materialite-du-genocide-des-tutsi// (consulté le 11 octobre 2021).

24 Périès Gabriel et Servenay David (2007), Une guerre noire. Enquête sur les origines du génocide rwandais (1959-1994), Paris, La Découverte ; Coret L. et Verschave F.-X., L'horreur qui nous prend au visage..., op. cit. ; Graner F., Le Sabre et la machette..., op. cit.

25 Dans la veine des débats sur les relations entre colonisateurs et colonisés, deux écueils semblent donc à éviter pour caractériser ces relations entre " coopérant » et " coopéré » au plan militaire : celui d'un coopérant omnipotent, commandant effectif mais occulte derrière des façades hiérarchiques locales, celui d'un coopérant impuissant et manipulé en permanence par ses homologues aux desseins machiavéliques dont il n'aurait rien su.

${ }^{26}$ Cette présence militaire française, si visible dans le paysage social de certains pays (Gabon, Djibouti, Tchad, Côte d'Ivoire, Sénégal), et abondamment étudiée sous l'angle des politiques de sécurité ou de la géopolitique, est pourtant très peu analysée dans sa longue durée par les sciences sociales : ses représentations, son épaisseur sociologique, sa " quotidienneté ", sa fabrique de l'exotisme, ses loisirs et son " ennui ", ses sociabilités et ses rencontres avec le pays de résidence, etc. Tout cet univers social est à reconstituer pour bien saisir les routines d'action et de pensée de ces militaires ensuite déployés en opération. Sur la genèse de la coopération militaire française en Afrique, voir par exemple Evrard Camille (2016), "Retour sur la construction des relations militaires franco-africaines ", Relations internationales, 165, pp. 23-42.

${ }^{27}$ Rapport Duclert, p. 167. Anatole Nsengiyumva, officier de renseignement et personnage clé de l'extrémisme CDR (Coalition pour la défense de la république, parti extrémiste hutu résolument opposé aux accords d'Arusha) sera d'ailleurs désigné officier de liaison de l'opération Turquoise. II connaît bien son interlocuteur français, Jacques Rosier, patron du COS.

28 Tauzin D., Rwanda..., op. cit., p. 71
} 
Une autre limite du rapport sur ces aspects militaires est la porte simplement entrouverte sur des pratiques « officieuses » ou «parallèles ». Tout le paradoxe de ce rapport qui s'est intéressé uniquement aux archives écrites des administrations publiques est pourtant d'avoir bien mis en évidence l'importance de «l'ordre par la voix ${ }^{29}$ ». Et d'avoir mis le doigt, en des termes forts, sur des pratiques pour le moins irrégulières, comme celles du général Huchon par exemple, dont certaines consignes «pourra[ient] s'apparenter à des pratiques d'officine ${ }^{30}$ ». Mais sans avoir approfondi la question. On touche là les limites d'une approche exclusivement archivistiques comme l'ont souligné les contributions d'historiens ${ }^{31}$ comme de militaires euxmêmes ${ }^{32}$.

Une des questions aussi laissée dans l'ombre par le rapport est le recours éventuel à des mercenaires, dans le cadre d'une stratégie indirecte ouvertement évoquée par le général Quesnot dans une note du 16 mai 199433. Qu'elle ait été tentée et qu'elle ait échoué à inverser le rapport de force, qu'elle ait été amorcée puis « terminée » en raison des risques médiatiques trop grands ou de la victoire plus rapide que prévue du FPR, ou qu'elle ait été simplement envisagée mais jamais réalisée, importe grandement : le jugement sur la responsabilité d'une éventuelle aventure mercenariale ne sera pas la même. Qu'elle ait porté sur l'entraînement, la fourniture de munitions, ou sur la réalisation directe d'objectifs militaires, le degré d'implication ne sera pas le même. Mais encore faut-il que les pièces du dossier soient sur la table. À cet égard, le rôle de Paul Barril et de Bob Denard a été évoqué et mériterait de plus amples recherches. Les intéressés eux-mêmes ne s'en sont pas cachés ${ }^{34}$. Or on dispose surtout de témoignages dans le cadre de procédure judiciaires ${ }^{35}$, de correspondances, de mentions de

\footnotetext{
${ }^{29}$ Au bout de la chaîne, le colonel Tauzin se félicite ainsi dans son ouvrage d'avoir reçu fin février 1993 « l'ordre le plus merveilleux que j'ai reçu de ma carrière militaire, un ordre oral » lors de l'opération Birunga destinée à contrer activement le FPR et pendant laquelle il commande indirectement les FAR. L'opération est restée secrète jusqu'en 1998. Tauzin D., Rwanda..., op. cit., p. 64.

30 Rapport Duclert, p. 753. Le rapport documente également comment le général Huchon et le général Quesnot déploient une rhétorique visant à empêcher tout rapprochement avec le FPR suite à la prise de Kigali par ce dernier, rhétorique faite de bellicisme contre I'ONU, de menaces de protestations de cadres dans l'armée si le pouvoir politique n'assume pas sa politique passée, de diabolisation à tout prix du FPR, qui annonce déjà le discours de l'association France-Turquoise dans le ton et les objectifs.

31 Robinet F., « Rwanda 1994... », art. cité.

32 Ce commentaire du général Patrice Sartre résume parfaitement cet enjeu : «Voulant tirer au clair les mécanismes de ce drame, le président Macron a mis les historiens au défi de l'ouverture (presque) totale des archives de l'État. Pour qui a vécu les événements analysés, la radiographie qui en résulte surprend, n'en présentant que les "parties dures", celles qui ont survécu par des écrits ou des enregistrements. Au contraire, les "parties molles" - les échanges verbaux, les connivences, les propos oubliés dans les procèsverbaux, sans parler des documents détruits, volontairement ou non - manquent évidemment à une image fidèle du passé ». Sartre P., « Le rapport Duclert... », art. cité.

33 Note du général Quesnot à l'attention de monsieur le président de la République, objet : entretien avec le chef de l'État intérimaire du Rwanda, 6 mai 1994, en ligne. URL : http://francegenocidetutsi.fr/fgtshowdoc.php?num=447 (consulté le 11 octobre 2021). Cette note, dans laquelle le général Quesnot reprend à son compte l'analyse des extrémistes hutus, évoque " un lobby tutsi » qui aurait son emprise jusque sur " une partie de notre appareil d'État » et conclue par la proposition de recourir à " des moyens et des relais d'une stratégie indirecte qui pourraient rétablir un certain équilibre » a fait couler beaucoup d'encre. II faudrait certes s'entendre sur ce que recouvre précisément cette notion de "stratégie indirecte ", qui peut prendre différents aspects, mais le fait même que la possibilité ait été formulée renseigne sur l'état d'esprit des faucons français.

34 C'est sur le site à la gloire de Bob Denard (URL: www.orbspatrianostra.com, consulté le 11 octobre 2021) qu'on trouve mention d'une mission menée au Rwanda sans précision des dates et objectifs exacts. Sur les activités présumées de Bob Denard au Rwanda, voir François Crétollier (dir.), "Le crapuleux destin de Robert-Bernard Martin. Bob Denard et le Rwanda », Montreuil, association Survie, février 2018, en ligne. URL : https://survie.org/publications/brochures/article/le-crapuleux-destin-de-robert-bernard-martinbob-denard-et-le-rwanda (consulté le 11 octobre 2021). Sur celles de Paul Barril, voir Perrin Jean-Pierre (2010), "Barril l'affreux », Revue XXI, 10, pp. 52-61. Voir également Dupaquier J.-F., Politiques, militaires..., op. cit.

35 Voir entre autres les témoignages de l'ancien Premier ministre du gouvernement intérimaire pendant le génocide, Jean Kambanda, sur les contrats passés par son gouvernement avec Paul Barril et Bob Denard, de l'ancien animateur de la Radio-télévision libre des mille collines (RTLM) Georges Ruggiu sur les mercenaires à Kigali puis Gisenyi, accompagnant les généraux Bizimungu et Kabiligi.
} 
contrats et des montants correspondants ${ }^{36}$. L'État n'ignorait du reste pas ces activités ${ }^{37}$, même si Mitterrand déclarait dans une étrange formule que "Barril n'est mandaté par personne ${ }^{38}$ ". Approfondir cette question n'est pas sans intérêt, tant du Katanga de 1961 au Rwanda de 1994 (puis au Zaïre en 1996-1997), l'image des mercenaires plane avec insistance et participe ainsi à façonner par leurs zones d'ombres et leurs silences les représentations et parfois les fantasmes sur la politique française en Afrique.

\section{La fin du lobby Turquoise? Pas si vite!}

S'il a permis de faire bouger les lignes en confortant les "modérés » au sein de l'armée française de l'époque, le rapport Duclert ne semble pas avoir permis de désamorcer le lobbying d'anciens militaires réunis dans l'association France-Turquoise ${ }^{39}$. Cette association en pointe sur la défense intransigeante du rôle de la France au Rwanda, et parallèlement dans la dénonciation du régime du FPR à Kigali, fut particulièrement active dans la médiatisation de "l'enquête du juge Bruguière », dans la promotion des ouvrages de Pierre Péan, pour in fine tenter de saborder la réconciliation franco-rwandaise mise en œuvre sous le quinquennat Sarkozy.

Avant et après la publication du rapport Duclert, ses principaux protagonistes ont réagi sur les canaux habituels, qui coïncident en grande partie avec ceux de l'extrême droite ou de la constellation souverainiste ${ }^{40}$. Début 2021, avant même la publication du rapport, le colonel de gendarmerie Michel Robardey, vice-président de France-Turquoise et plume prolixe, rediffusait sa préface écrite en 2018 de l'ouvrage d'Hervé Cheuzeville. Cette préface approuve la démonstration proposée par l'auteur selon laquelle le FPR a « délibérément provoqué » le génocide des Tutsi $^{41}$. Une fois le rapport publié, l'association s'est félicitée officiellement de la mise à l'écart de toute notion de complicité dans le génocide des Tutsi en publiant les communiqués de Jean-Claude Lafourcade, président de l'association, et de la ministre des Armées visant à rassurer les militaires ${ }^{42}$. Pourtant, suite à cette réaction officielle et convenue, le site de l'association n'a pas tardé à faire son miel de textes critiques contre le rapport comme ceux de Jacques Hogard entre autres ${ }^{43}$.

\footnotetext{
36 "A Foreseeable Genocide. The Role of the French Government in Connection with the Genocide Against the Tutsi in Rwanda », Washington, Levy Firestone Muse LPP, 19 avril 2021, pp. 368-371, en ligne. URL: https://www.gov.rw/fileadmin/user_upload/gov_user_upload/2021.04.19_MUSE_REPORT.pdf (consulté le 11 octobre 2021).

37 Dans l'inventaire des fiches de la DGSE (Direction générale de la Sécurité extérieure) sur le Rwanda répertoriées par la commission Duclert figurent trois notes sur les activités de Paul Barril et de Bob Denard : Fiche $n^{\circ}$ 19123/N du 26 juillet 1994 : « Activités de Bob Denard »; Fiche n 19149 du 29 juillet 1994 : «Projets du capitaine Barril »; Fiche n 19273/N du 22 août 1994 : «Poursuite des activités de Bob Denard ». Rapport Duclert, pp. 1195-1196.

38 Saint-Exupéry P. (de), L'Inavouable..., op. cit., p. 256.

39 Cette association créée en 2007 en réaction aux accusations de complicité de génocide portées contre l'armée française est née d'un blog du colonel Jacques Hogard destiné à faire la promotion de son livre (URL : http://rwanda.viabloga.com), suspendu puis ensuite transformé en association avec son site internet (et présidée par le général Lafourcade). Ses membres les plus actifs en ligne furent et sont Jacques Hogard et Michel Robardey. URL : https://www.france-turquoise.com/ (consulté le 11 octobre 2021).

40 Radio Courtoisie, TV Libertés, Spoutnik France, Valeurs actuelles, Marianne.

41 Dans la version initialement publiée, cette note portait une appréciation sur les propos de M. Robardey insuffisamment fondée que nous avons pris la décision de retirer.

42 Voir Communiqué du général Lafourcade et lettre de la ministre des Armées au général Lafourcade, 27 et 29 mars 2021 , en ligne. URL : https://www.france-turquoise.com/rapport-duclert-deux-reactions/ (consulté le 11 octobre 2021).

43 Voir par exemple la réaction le 13 avril 2021 du colonel Hogard interrogé sur la chaîne internet TV Libertés, présenté comme « acteur majeur de l'opération Turquoise » et « homme de terrain », devenu " expert » de l'ensemble du dossier rwandais de 1990 jusqu'aux événements du Congo... (en ligne. URL: https://www.tvlibertes.com/passe-present-n302-avec-le-colonel-hogard-les-
} 
L'association France-Turquoise est épaulée dans son combat par Secours de France, association catholique proche de l'extrême droite et des nostalgiques de l'Algérie française. Secours de France a ainsi décerné son prix 2019 Clara Lanzi à France-Turquoise et relaie également les interventions de Bernard Lugan, Jacques Hogard et Hubert Védrine sur le sujet du Rwanda. La rubrique de son site intitulée «Falsifications de I'histoire » ne fait pas mystère de sa volonté d'étriller le rapport Duclert (et le rapport Stora) sans aucun sens de la nuance ${ }^{44}$. Une autre association, I'Association de soutien à l'armée française (ASAF), relaie également les positions de France-Turquoise. L'ASAF s'est fendue d'un "Dossier Rwanda », publié en avril 2019 à l'occasion des 25 ans du génocide ${ }^{45}$. On y retrouve les protagonistes habituels, Michel Robardey et Jacques Hogard de France-Turquoise, ainsi que l'inévitable Hubert Védrine.

II est facile de constater à quel point, dans ces segments qu'il faut bien qualifier d'extrémistes, on est loin d'une remise en question de l'engagement français au Rwanda. Pour ce réseau, la publication du rapport Duclert ne change pas la donne mais vient confirmer l'idée que, comme sous le quinquennat Sarkozy avec le ministère Kouchner, le pouvoir politique voudrait sacrifier l'honneur de l'armée au profit d'une réconciliation avec Kigali imposée par la géopolitique. Sur cette position retranchée, on retrouve le même réseau d'acteurs depuis 25 ans, qui tente de verrouiller les interrogations critiques sur le rôle de la France au Rwanda. C'est un réseau de plus en plus minoritaire et isolé, qui lit et s'informe en vase $\operatorname{clos}^{46}$, mais un réseau néanmoins très actif, au point qu'à chaque fois qu'un président français a voulu œuvrer à une réconciliation franco-rwandaise, le lobby France-Turquoise s'est mis au travers du chemin, sous la présidence Chirac, sous Sarkozy, sous Macron enfin. Mais la partie semble leur échapper désormais, et leur récit tend de plus en plus à devenir une contre-mémoire.

Il faut souligner, en effet, qu'un des effets indirects du rapport Duclert a été de permettre la rencontre entre d'anciens militaires français et le président Paul Kagame en mai 2021 à

\footnotetext{
reponses-sur-le-genocide-rwandais, consulté le 11 octobre 2021) ou son interview le 27 mai à Boulevard Voltaire dans laquelle il accuse Vincent Duclert d'être «tout sauf historien, mais plutôt politique, partiel et partial » (en ligne. URL: https://www.bvoltaire.fr/colonel-jacques-hogard-la-france-est-accusee-or-le-genocide-du-rwanda-a-ete-commis-par-des-rwandaiscontre-des-rwandais/, consulté le 11 octobre 2021). Parmi les réactions signalées sur le site de l'association, voir aussi la réaction du général Michel Fruchard, ancien des troupes de marine et membre du cabinet militaire du ministre de la Défense de 1990 à 1993 , qui ne passe pourtant pas dans le rapport pour un radical : Michel Fruchard, "Rapport Duclert : les dés étaient pipés », Espritsurcouf, 18 avril 2021, en ligne. URL : https://espritsurcouf.fr/humeurs_rapport-duclert-les-des-etaient-pipes_par_le-general-michel-fruchard/ (consulté le 11 octobre 2021).

44 L'analyse du rapport Duclert dans cette rubrique est signée Bernard Lugan, personnage bien connu dans les milieux militaires et à l'extrême droite. Ayant enseigné au Rwanda à la fin des années 1970 et au début des années 1980, il passe pour spécialiste du Rwanda aux yeux de générations d'officiers qui l'ont eu comme enseignant dans les formations d'officiers (Saint Cyr et l'Institut des hautes études de défense nationale notamment). Ses ouvrages sont autant de " bibles » pour officiers supérieurs et généraux d'une certaine génération. II joua un rôle efficace de filtre sur les savoirs africanistes transmis dans les armées, au profit d'une lecture extrêmement datée du continent. Pour ce nostalgique de la France coloniale et des beaux jours de la Françafrique, le FPR joue le rôle de nouveau Front de libération nationale (FLN), et toute interrogation critique sur l'histoire de France range son auteur dans la catégorie de « traître ».

45 Association de soutien à l'armée française, «Dossier Rwanda », avril 2019, en ligne. URL: https://www.asafrance.fr/images/rwanda_dossier_asaf_070419.pdf (consulté le 11 octobre 2021).

46 Les suggestions bibliographiques de l'association France-Turquoise sur son site sont édifiantes à cet égard.
} 
Paris $^{47}$, chose inconcevable jusqu'alors ${ }^{48}$. Un jour prochain, peut-être, d'anciens militaires français assisteront aux commémorations du génocide à Kigali. Ce jour-là, un grand pas aura été franchi. Le syndrome rwandais de l'armée française aura été véritablement dépassé.

Étienne Smith

Les Afriques dans le monde, Sciences Po Bordeaux (France)

\section{Bibliographie}

Ambrosetti David (2001), La France au Rwanda. Un discours de légitimation morale, Paris, Karthala.

AnCEL Guillaume (2018), Rwanda, la fin du silence. Témoignage d'un officier français, Paris, Les Belles Lettres.

CHREtIEN Jean-Pierre (2009), « France et Rwanda : le cercle vicieux », Politique africaine, 113, pp. 121137.

Collombat Benoît et Servenay David (2014), "Au nom de la France ». Guerres secrètes au Rwanda, Paris, La Découverte.

COMMISSION DE RECHERCHE SUR LES ARCHIVES FRANÇAISES RELATIVES AU RWANDA ET AU GENOCIDE DES TUTSI (2021), La France, le Rwanda et le génocide des Tutsi (1990-1994), Paris, Armand Colin.

CORET Laure et VersChAVE François-Xavier (dir.) (2005), L'Horreur qui nous prend au visage: l'État français et le génocide au Rwanda. Rapport de la Commission d'enquête citoyenne, Paris, Karthala.

Dallaire Roméo (2003), J'ai serré la main du diable. La faillite de l'humanité au Rwanda, Outremont (Québec), Libre Expression.

DeLoRT Dominique (2021), Guerre au Rwanda. L'espoir brisé, 1991-1994, Paris, Perrin/ Pierre de Taillac.

DoRIDANT Raphaël et Graner François (2020), L'État français et le génocide des Tutsis au Rwanda, Marseille, Agone.

DUPAQUIER Jean-François (2014), Politiques, militaires et mercenaires français au Rwanda. Chronique d'une désinformation, Paris, Karthala.

EVRARD Camille (2016), « Retour sur la construction des relations militaires franco-africaines », Relations internationales, 165 , pp. 23-42.

GRANER François (2014), Le Sabre et la machette: officiers français et génocide tutsi, Mons, Éditions Tribord.

\footnotetext{
47 Étaient présents, Éric de Stabenrath, ancien collègue de formation de Kagame à Fort Leavenworth en 1990, Jean Varret et René Galinié, déjà évoqués, ainsi que I'ancien ambassadeur en poste en Ouganda, Yannick Gérard. Mehdi Ba, « Rencontre entre Paul Kagame et d'ex-officiers français, nouvelle étape du rapprochement entre Paris et Kigali », Jeune Afrique, 19 mai 2021, en ligne. URL : https://www.jeuneafrique.com/1174620/politique/rencontre-entre-paul-kagame-et-dex-officiers-francais-nouvelle-etape-durapprochement-entre-paris-et-kigali/ (consulté le 11 octobre 2021).

48 Loin des caméras, des rencontres entre officiers français et rwandais ont également eu lieu sur divers théâtres d'opérations multinationales de maintien de la paix comme en République démocratique du Congo, au Mali ou en République centrafricaine ces dernières années.
} 
Hogard Jacques (2005), Les Larmes de l'honneur. 60 jours dans la tourmente du Rwanda, Paris, Hugo Doc.

LAFOURCADE Jean-Claude et Riffaud Guillaume (2010), Opération Turquoise. Rwanda 1994, Paris, Perrin.

LANOTTE Olivier (2007), La France au Rwanda (1990-1994). Entre abstention impossible et engagement ambivalent, Bruxelles, Peter Lang.

LARCHER Laurent (2019), Rwanda, ils parlent. Témoignages pour l'histoire, Paris Seuil.

MAS Monique (1999), Paris-Kigali 1990-1994. Lunettes coloniales, politique du sabre et onction humanitaire pour un génocide en Afrique, Paris/Montréal, L'Harmattan.

Peries Gabriel et Servenay David (2007), Une guerre noire. Enquête sur les origines du génocide rwandais (1959-1994), Paris, La Découverte.

PERRIN Jean-Pierre (2010), «Barril I'affreuX », Revue XXI, 10, pp. 52-61.

PRUNIER Gérard ([1995] 1999), Rwanda : le génocide, Paris, Dagorno.

ROBINET François (2014), "L'empreinte des récits médiatiques: mémoires françaises du génocide des Tutsi du Rwanda », Les Temps modernes, 680-681, pp. 166-188.

(2021), "Le rôle de la France au Rwanda: I'histoire piégée ? », Revue d'histoire culturelle. $X V_{I I}{ }^{P}-X X F^{f}$ siècles, 2 , en ligne. URL : https://revues.mshparisnord.fr/rhc/index.php?id=690 (consulté le 11 octobre 2021).

(2021), « Rwanda 1994 : un rapport pour I'histoire ? », Études, juillet-août, pp. 7-18.

SAINT-EXUPERY Patrick (de) (2004), L'Inavouable. La France au Rwanda, Paris, Les Arènes.

SMITH Étienne (2014), «Les derniers défenseurs de l'empire: quand l'armée française raconte ses Rwanda », Les Temps modernes, 680-681, pp. 66-100.

TAUZIN Didier (2011), Rwanda : je demande justice pour la France et ses soldats. Le chef de l'opération Chimère témoigne, Paris, Jacob Duvernet.

VAlleE Shahin (2021), «Rapport Duclert sur le génocide des Tutsi : un pas de plus », Le Grand Continent, 2 avril, en ligne. URL: https://legrandcontinent.eu/fr/2021/04/02/rapport-duclert-sur-le-rwanda-unpas-de-plus/ (consulté le 11 octobre 2021).

VARRET Jean (2018), Général, j'en ai pris pour mon grade, Paris, Éditions Sydney Laurent.

VULPIAN Laure (de) et PRUNGNAUd Thierry (2012), Silence Turquoise. Rwanda 1992-1994, responsabilités de l'État français dans le génocide des Tutsi, Paris, Don Quichotte. 